\title{
Participación de MT1-MMP en la Remodelación del Ligamento Periodontal Durante la Movilización Dentaria
}

\author{
Role of MT1-MMP in the Remodeling of the Periodontal Ligament \\ During Tooth Movement
}

Rey Droghetti $\mathrm{P}^{1}$, Cruzat $\mathrm{F}^{2}$, Smith Ferrer $\mathrm{P}^{3}$, Oyarzún Droguett $\mathrm{A}^{4}$

\begin{abstract}
RESUMEN
La movilización dentaria involucra una serie de cambios en los tejidos de soporte caracterizados por la activa remodelación de estos. La MT1-MMP o MMP-14 es una potente enzima proteolítica capaz de degradar colágeno tipo I, la principal molécula estructural del ligamento periodontal. La migración dentaria requiere de la degradación controlada del colágeno constituyente del ligamento periodontal. Sin embargo, no existen evidencias de la participación de MT1-MMP en la remodelación del tejido periodontal durante este proceso. En el presente estudio hemos analizado la expresión de MT1-MMP y del marcador de actividad osteoclástica Fosfatasa Ácida Tartrato Resistente (TRAP) en un modelo de migración dentaria en ratas. La migración dentaria fue activada mediante la inserción de una banda separadora entre los incisivos superiores. La expresión y distribución de TRAP y MT1-MMP fue evaluada a través de citoquímica e inmunohistoquímica a los días 1, 3,5 y 7 . La producción de TRAP fue identificada principalmente en osteoclastos ubicados en la zona de compresión del ligamento periodontal. La producción de MT1-MMP fue observada en fibroblastos de la zona de compresión del ligamento periodontal y osteoclastos ubicados en esta misma región. Nuestros resultados permiten proponer que tanto MT1-MMP como TRAP participan en la remodelación de los tejidos de soporte periodontal durante la migración dentaria.
\end{abstract}

Rev. Clin. Periodoncia Implantol. Rehabil. Oral Vol. 3(3); 113-117, 2010.

Palabras clave: MT1-MMP, remodelación tisular, ligamento periodontal, osteoclasto, movimiento dentario.

\section{ABSTRACT}

Tooth movement involves a series of changes of the supporting periodontal tissues characterized by the active connective tissue remodeling. MT1MMP or MMP-14 belongs to the family of matrix metalloproteinases that are able to degrade type I collagen, the main molecule involved in periodontal attachment. Tooth migration requires the controlled degradation of periodontal ligament collagen fibers. However, evidences linking MT1-MMP expression with periodontal tissue remodeling are lacking. In the present study, we have evaluated the expression of MT1-MMP and of the osteoclast marker Tartrate Resistant Acid Phosphatase (TRAP) in a model of tooth migration in rats. Tooth migration was induced after the insertion of a rubber band between the upper incisors. The distribution of TRAP and MT1-MMP was evaluated by means of cytochemistry and immunohistochemistry respectively at days 1, 3, 5 and 7. TRAP production was identified in osteoclasts at the area of compression of the periodontal ligament. MT1-MMP distribution was observed in fibroblasts at the compressed area of the periodontal ligament and also in osteoclasts of the same region. Our findings allow us to propose that MT1-MMP and TRAP take part of the tissue remodeling events observed during tooth movement.

Rev. Clin. Periodoncia Implantol. Rehabil. Oral Vol. 3(3); 113-117, 2010.

Key words: MT1-MMP, tissue remodeling, periodontal ligament, osteoclast, tooth movement.

\section{INTRODUCCIÓN}

La movilización dentaria implica la activación de una serie de cambios adaptativos en los tejidos periodontales que permiten el desplazamiento de los dientes a través del proceso alveolar y finalmente la reconstitución de un periodonto funcional. Para esto, es necesario que ocurra una activa remodelación en los tejidos de inserción periodontal, y por lo tanto de las moléculas constituyentes de la matriz extracelular $(\mathrm{MEC})^{(1)}$. La aplicación de fuerzas sobre una pieza dentaria genera una serie de cambios tisulares en el tejido de soporte periodontal. En la zona del periodonto sometida a una fuerza compresiva es posible identificar una activa degradación del tejido conectivo y óseo. La remodelación de este componente tisular es llevada a cabo por osteoclastos que derivan de precursores presentes en la médula ósea hematopoyética ${ }^{(2,3)}$. Por otro lado, en la zona de tracción se distingue la aposición de tejido óseo por la actividad de osteoblastos presentes en el tejido(1). Esta combinación de síntesis y degradación de matriz es fundamental para lograr un desplazamiento equilibrado de las piezas dentarias en los huesos maxilares.

La remodelación de la MEC es ejecutada a través de diferentes cascadas de proteolíticas en que participan una amplia familia de enzimas $^{(4,5)}$. Estas corresponden a la vía de las metaloproteasas de la

1. Cirujano Dentista. Integramédica. Estudiante Postítulo en Periodoncia, Universidad Mayor. Chile.

2. Cirujano Dentista. Centro Médico San Joaquín. Pontificia Universidad Católica de Chile. Chile.

3. Carrera de Odontología. Facultad de Medicina, Pontificia Universidad Católica de Chile. Chile.

4. Facultad de Odontologia. Universidad Finis Terrae. Chile.

Correspondencia autor: Patricio Smith Ferrer. psmith@med.puc.cl. Laboratorio de Fisiología Periodontal, Carrera de Odontología. Facultad de Medicina, Pontificia Universidad Católica de Chile. Marcoleta 391, Santiago. Chile.

Financiamiento: Proyecto de Investigación FONDECYT No 1061065 (PS). Trabajo recibido el 01/11/2010. Aprobado para su publicación el $27 / 12 / 2010$. 
matriz (MMPs), la vía del plasminógeno, vía fagocítica y vía osteoclástica(5). En el caso de la remodelación periodontal que ocurre durante el movimiento dentario, existen evidencias que varias de estas cascadas se encuentran activas o reguladas positivamente y se presume que participan en la degradación de componentes de la MEC periodontal(6-10)

Las metaloproteasas de la matriz (MMPs) o matrixinas corresponden a enzimas proteolíticas que participan en la remodelación de los tejidos conectivos a través del procesamiento proteolítico, tanto de moléculas estructurales de la matriz extracelular, como de citoquinas y factores de crecimiento que regulan diferentes actividades celulares ${ }^{(4,5)}$. Las MMPs son producidas por una amplia variedad de células durante la remodelación tisular observada durante el desarrollo embrionario, enfermedades inflamatorias, patologías degenerativas articulares, invasión tumoral y reparación de heridas ${ }^{(5)}$. La mayoría de ellas son secretadas como pro-enzimas, las cuales deben ser activadas a través del procesamiento proteolítico de su prodominio y la consecutiva exposición de su dominio catalítico(5). Finalmente, la actividad catalítica de estas proteasas es bloqueada por inhibidores específicos conocidos como inhibidores titulares de metaloproteasas TIMPs ${ }^{(5)}$.

El ligamento periodontal está conformado principalmente por colágeno tipo I y III, siendo su principal función la sujeción mecánica entre la superficie dentaria y el hueso alveolar ${ }^{(11)}$. Durante el movimiento dentario, este tejido conectivo debe ser remodelado. La degradación proteolítica del colágeno fibrilar (tipo I y III) sólo puede ser ejecutada por un grupo restringido de enzimas proteolíticas conocidas como colagenasas $^{(5)}$. Se ha demostrado que el bloqueo farmacológico de la actividad de las MMPs es capaz de interferir en el movimiento dentario en animales de experimentación, lo que demuestra la importancia de esta familia de enzimas en el fenómeno de movilización dentaria (Bildt et al., 2007). Dentro de las MMPs capaces de degradar colágeno fibrilar se incluyen la MMP-1, MMP-8, MMP-13 y MT1-MMP (MMP-14)(5). La participación de algunas de estas proteasas, tales como MMP-1, -8 y -13 , ha sido documentada durante el movimiento dentario ortodóncico en modelos animales y/o humanos ${ }^{(7,8,9,12)}$. La MT1-MMP corresponde a una metaloproteasa de transmembrana que posee actividad catalítica contra diferentes sustratos tales como colágeno tipo I, II y III, fibronectina, laminina 1 y 5, vitronectina, proteoglicanos (CD-44), Factor de Necrosis Tumoralalfa (TNF- $\alpha$ ) y Factor de Crecimiento de Tejido Conectivo (CTGF) ${ }^{(13,14)}$. La MT1-MMP puede a su vez activar a la pro-MMP-13(13) y pro-MMP-2 a nivel de la superficie celular, y participa en la formación de un complejo trimolecular en conjunto con el TIMP-2 y la forma inactiva de MMP-2 ${ }^{(13)}$. La expresión de MT1-MMP ha sido identificada recientemente en los tejidos periodontales comprometidos por la enfermedad periodontal, en los cuales se ha identificado un incremento en la expresión de esta enzima y un desbalance entre la proteasa y su inhibidor TIMP-2. En este mismo estudio se pudo evidenciar la colocalización de MT1-MMP con fibras de colágeno del tejido gingival, resultado que refuerza el papel de la proteasa en la remodelación del colágeno en esta enfermedad(15). El TIMP-2 actúa como el principal inhibidor de la actividad proteolítica derivada de MT1$\mathrm{MMP}^{(13)}$. Una herramienta de gran utilidad en la identificación del papel que juegan estas proteasas en la remodelación tisular es el empleo de ratones knock-out. Es destacable que el ratón deficiente (knock-out) en MT1-MMP manifiesta una serie de alteraciones en la degradación de colágeno fibrilar, lo que pone en evidencia la importancia de esta enzima proteolítica en la remodelación del colágeno fibrilar ${ }^{(16)}$. Esta alteración en la producción de MT1-MMP tiene importantes efectos en el crecimiento y desarrollo del esqueleto en general y en el desarrollo dentario y radicular ${ }^{(7,13)}$. Los osteoclastos son células multinucleadas encargadas de la reabsorción ósea que se diferencian in situ a partir de precursores de origen monocítico ${ }^{(17)}$. Las estructuras más abundantes en su citoplasma son los lisosomas, vesículas ricas en enzimas como la fosfatasa ácida. En el hueso se pueden identificar dos tipos de fosfatasa ácida. Ambas formas han sido caracterizadas bioquímicamente con respecto a su especificidad frente a diferentes sustratos y pH óptimo. Estas enzimas a su vez responden funcionalmente a hormonas osteotrópicas in vitro. La Fosfatasa Ácida Tartrato Sensible ha sido localizada en osteoblastos, osteocitos y osteoclastos, mientras que la Fosfatasa Ácida Tartrato Resistente (TRAP) se ubica únicamente en osteoclastos, y ha demostrado ser un marcador citoquímico para estas células ${ }^{(18)}$. Es importante destacar que, por un mecanismo aún no clarificado, la deficiencia en la expresión de MT1-MMP en el ratón knock-out para esta enzima se acompaña de un incremento en la expresión de la enzima TRAP y un incremento asociado en la diferenciación osteoclástica ${ }^{(16)}$. Esto sugiere que la producción de ambas enzimas se encuentra de alguna forma coordinada y que la MT1-MMP puede regular la diferenciación osteoclástica. Para permitir la migración de los pre-osteoclastos y la formación de una laguna reabsortiva, es necesario que ocurra la remoción de matriz orgánica del tejido óseo. Recientemente, diferentes estudios han demostrado la participación activa de una serie de proteasas, entre ellas catepsina $\mathrm{K}$ y MT1-MMP, durante la migración y anclaje de los osteoclastos, localizándose esta última principalmente en las evaginaciones de la membrana celular o podosomas ${ }^{(19)}$. Se ha observado también que MT1-MMP es sobreexpresada en la superficie de células tumorales, donde su actividad proteolítica facilitaría el crecimiento e invasividad de las células tumorales a través de la MEC ${ }^{(20)}$. El presente estudio fue diseñado para probar la hipótesis que TRAP y MT1-MMP participan en la remodelación tisular que ocurre durante la movilización dentaria.

\section{MATERIALES Y MÉTODO}

\section{Modelo Experimental y Procesamiento de la Muestra}

Se utilizaron 10 ratas Sprague-Dawley machos, adultas, de aproximadamente 200 grs. Los procedimientos para el manejo de animales fueron aprobados por el Comité de Ética de la Facultad de Odontología de la Universidad de Chile. Los animales fueron anestesiados con Tiopental Sódico $1 \mathrm{gr}$ (Bestpharma S.A.) al $0.5 \%$, inyectado intraperitonealmente. A estos animales se les insertó entre los incisivos centrales superiores una banda elástica (Unitek TM Elastics, $3 \mathrm{M}$, Bummer, 3/16", Heavy, $6 \mathrm{oz}$ ) que fue fijada utilizando una resina compuesta del Sistema Single Bond (3M, St Paul, MN, USA). Dos especimenes, que sirvieron como control, no recibieron la mencionada banda. Durante el período postoperatorio los especímenes fueron alimentados ad libitum y monitoreados periódicamente. Se evitó el empleo de anti-inflamatorios por el impacto que este tipo de fármacos puede tener en la remodelación ósea y de tejidos conectivos. Todos los animales sobrevivieron al procedimiento y mostraron una recuperación satisfactoria. Los especímenes experimentales fueron sacrificados a los 1, 3, 5 y 7 días consecutivos a la instalación de la banda elástica mediante una sobredosis intraperitoneal de Tiopental Sódico. Esto permitió completar un total de dos animales por cada tiempo experimental para cada tiempo evaluado (1, 3, 5, 7 días) y dos animales controles que no recibieron la banda elástica. Inmediatamente después del sacrificio, los maxilares fueron removidos en bloque e inmersos en una solución fijadora de formaldehído al $10 \%$ tamponeado a pH 7.4 a $4^{\circ} \mathrm{C}$ durante 48 horas. Luego las muestras fueron descalcificadas en una solución de EDTA al $10 \%$ a $\mathrm{pH} 7.4$ a $4^{\circ} \mathrm{C}$ durante 30 días.

\section{Procesamiento Histológico}

Los especímenes se deshidrataron en etanoles de concentración ascendente, aclarados en xilol e incluidos en parafina. Se obtuvieron cortes seriados de $6 \mu \mathrm{m}$ de grosor, los que se montaron en portaobjetos xilanizados.

\section{Detección Citoquímica de TRAP}

Cortes de $6 \mu \mathrm{m}$ de los especímenes, experimentales y control, se incubaron en un medio para la detección citoquímica de TRAP. Se disolvieron $4 \mathrm{mg}$ de Naftol AS-MX (Sigma, St. Louis, MO) en $0.25 \mathrm{ml}$ de N, N-dimetilformamida. Posteriormente se agregó $25 \mathrm{ml}$ de buffer acetato $0.2 \mathrm{M}$ (pH 5.0) $30 \mathrm{mg}$ de Fast Red como agente de acoplamiento. Como control, el sustrato fue omitido. Luego se agregó $0.19 \mathrm{grs}$ de ácido tartárico $\mathrm{L}(+)$. Los cortes se incubaron durante 30 minutos a $37^{\circ} \mathrm{C}$ y luego fueron lavados en agua destilada.

\section{Detección por Inmunohistoquímica de MT1-MMP}

Los cortes fueron desparafinados en xilol e hidratados en PBS. Se realizó el desenmascaramiento antigénico con buffer Tris- $\mathrm{HCl}$ a $0.2 \%$, a pH 8 durante 2 ciclos de 5 minutos cada uno con un microondas convencional en su máxima potencia. Posteriormente, se bloqueó la peroxidasa endógena en peróxido de hidrógeno $\left(\mathrm{H}_{2} \mathrm{O}_{2}\right)$ al $3 \%$, durante 30 minutos. Los sitios inespecíficos fueron bloqueados con suero normal de cabra al $5 \%$ en PBS-BSA al $3 \%$ durante 30 minutos. Los cortes se incubaron durante 12 horas a $4^{\circ} \mathrm{C}$ en un anticuerpo monoclonal anti MT1-MMP humana (Calbiochem Anti MT1-MMP) en una concentración de $15 \mu \mathrm{g} / \mathrm{ml}$. La inmunoreacción se detectó utilizando el sistema Biotina -Streptavidina-Peroxidasa-Diaminobenzidina (Histostain Plus). Como 
control, algunos cortes se incubaron en suero de cabra no inmune. Se realizó una tinción de contraste nuclear con hematoxilina de Harris y con Fast Green FCF al 0.1\%.

\section{RESULTADOS}

La inserción de una banda elástica entre los incisivos superiores indujo la movilización dentaria. En la Figura 1A se muestra que la aplicación de este procedimiento logró una movilización dentaria efectiva al cabo de 7 días. A la observación microscópica, y mediante la prueba citoquímica para la detección de TRAP, se evidenció una fuerte marcación para esta enzima en el área de presión y una marca débil en el área de tensión en los especímenes experimentales (Figura 1B). Todos los animales sometidos a este experimento sobrevivieron adecuadamente el procedimiento. El análisis secuencial de la expresión de TRAP a través del tiempo permitió detectar una fuerte marca para esta enzima en el área de presión del ligamento periodontal, siendo la

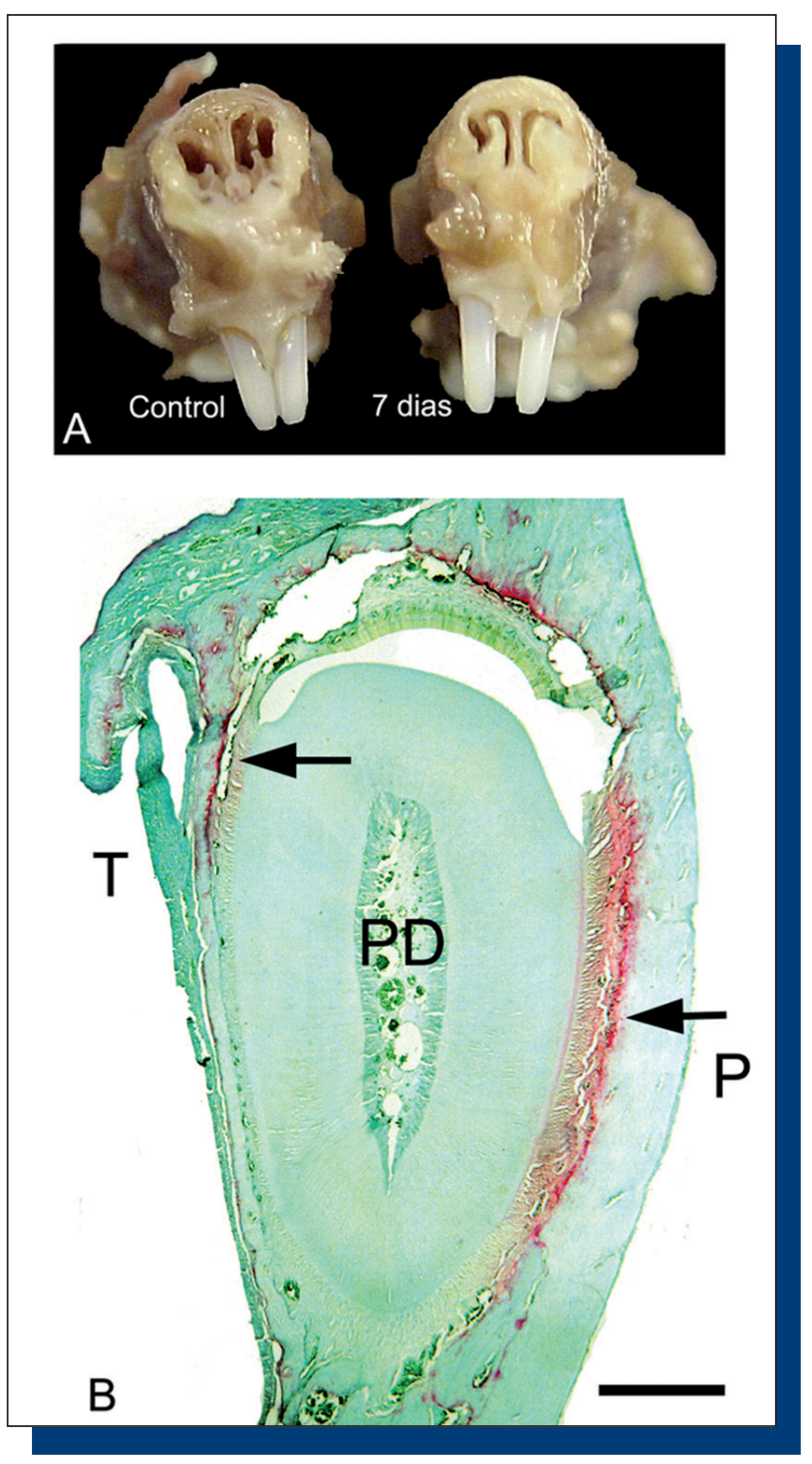

Figura 1. Desplazamiento dentario y expresión de TRAP. A: Visión frontal de los incisivos superiores que muestra la separación dentaria lograda luego de 7 días de inserción de una banda elástica. B: Corte transversal de un incisivo central de un espécimen experimental sacrificado al día 7 y procesado mediante citoquímica para revelar la expresión de TRAP (flechas). T: Lado de tensión del ligamento periodontal. P: Lado de presión del ligamento periodontal. PD: Pulpa dental. Barra: $250 \mu \mathrm{m}$. marca en el área de tensión de mucho menor intensidad en todos los tiempos examinados (Figura 2). A nivel morfológico se pudo observar que el área de tensión presentó, además de una menor marca para TRAP, un contorno lineal del hueso alveolar (Figura 3A). Esto fue claramente diferente en el área de compresión, en la cual destacó la intensa marca para TRAP en asociación con la presencia de lagunas de reabsorción compatibles con la actividad de osteoclastos (Figura 3B).

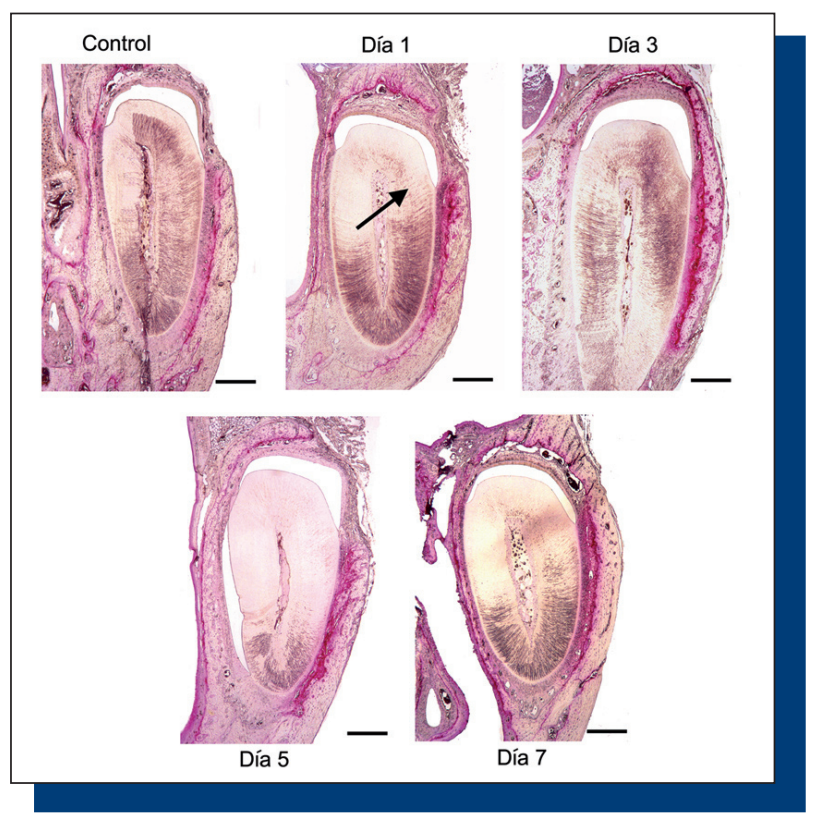

Figura 2. Expresión de TRAP durante la movilización dentaria. Corte transversal de dientes control y experimental a los días $1,3,5$ y 7 procesados mediante citoquímica para la expresión de TRAP. La flecha indica la dirección del movimiento. Obsérvese la mayor intensidad en la marca para TRAP en el área de compresión del ligamento periodontal. Barra: $250 \mu \mathrm{m}$.

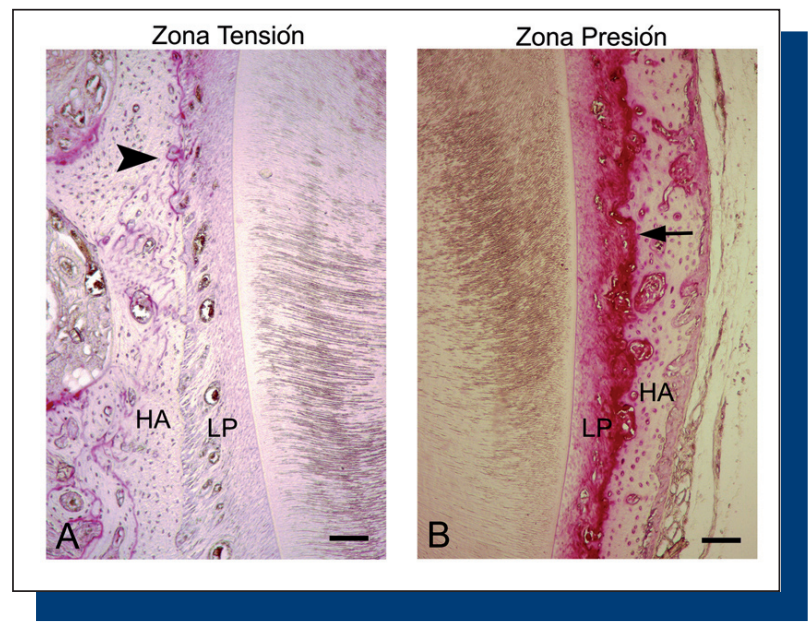

Figura 3. Expresión diferencial de TRAP en zonas de presión y tinción del ligamento periodontal. Comparación de zonas de presión y tensión en un corte experimental del día 3 procesado mediante citoquímica para TRAP. A: Lado de tensión. Se observa una reducida expresión de la marca para TRAP (cabeza de flecha) en el área del ligamento periodontal (LP) próxima al hueso alveolar (HA). Barra: $50 \mu \mathrm{m}$. B: Lado de presión. Se puede observar una fuerte marca para TRAP distribuida en el ligamento periodontal (LP) y hueso alveolar (HA). Barra: $50 \mu \mathrm{m}$.

La inmunomarcación para MT1-MMP fue observada fundamentalmente en las áreas de compresión de los tejidos periodontales. Esta marca fue detectada principalmente en osteoclastos multinucleados y algunas células mononucleadas del ligamento periodontal vecino a las zonas de reabsorción ósea compatibles con fibroblastos (Figura 4A, B y C). El reemplazo del anticuerpo primario por suero no inmune, control negativo, no mostró inmunomarcación (Figura 4D). 


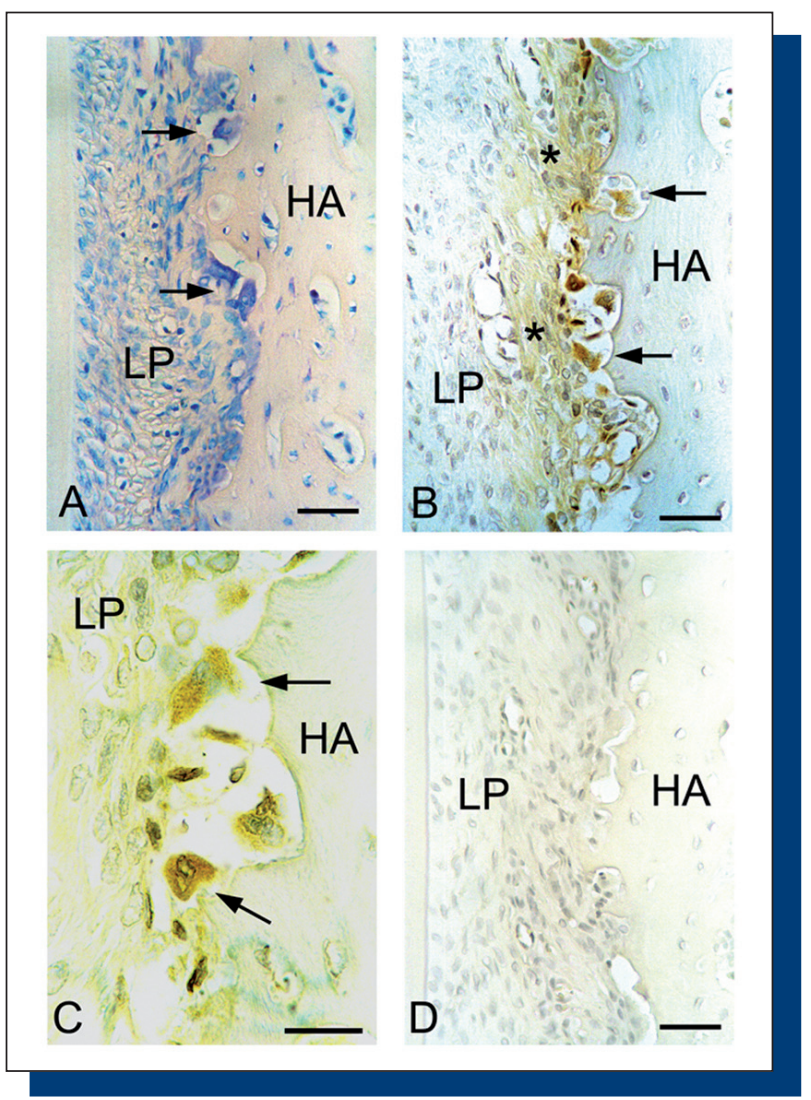

Figura 4. Expresión de MT1-MMP en el área de presión generada por la movilización dentaria. A: Zona de presión, donde se observa la presencia de osteoclastos (flecha) en la interfase entre el hueso alveolar (HA) y ligamento periodontal (LP). Tinción: Giemsa. Barra: $50 \mu \mathrm{m}$. B: Inmunoreacción para MT1MMP en color café (asterisco), y la marca en relación con los osteoclastos (flecha). Barra: $50 \mu \mathrm{m}$. C: Osteoclastos positivos para MT1-MMP (flechas). Barra: $50 \mu \mathrm{m}$ D: Control negativo, en el que se aprecia ausencia de marca para MT1-MMP. Barra: $50 \mu \mathrm{m}$.

\section{DISCUSIÓN}

La movilización dentaria implica la activa remodelación del tejido periodontal y óseo adyacente a la pieza dentaria. A nivel histológico dos zonas pueden ser reconocidas en los tejidos periodontales, un área de reabsorción ubicada en la zona de compresión y otra de aposición ósea en la zona de tracción ${ }^{(1)}$. En ambas áreas existe una activa remodelación de los tejidos periodontales, lo que implica la expresión de proteasas capaces de degradar macromoléculas estructurales de la MEC y el procesamiento proteolítico de factores de crecimiento y citoquinas que modulan de manera crítica diferentes respuestas celulares, tales como diferenciación, migración, adhesión, proliferación y muerte celular ${ }^{(1)}$. En el presente estudio hemos analizado la expresión de dos importantes enzimas involucradas en la remodelación tisular como son TRAP y MT1-MMP durante el movimiento dentario. Para la realización de este experimento se utilizó un modelo de movilización dentaria en ratas, en las que se provocó la separación de los incisivos centrales superiores mediante la inserción de una banda elástica entre ambos dientes. Posterior a la ejecución del procedimiento antes descrito, las ratas fueron monitoreadas y sacrificadas transcurridos 1, 3, 5 y 7 días luego de la inserción de la banda. Se pudo constatar que el modelo de movilización dentaria fue exitoso, ya que se observó la separación entre los incisivos superiores. A nivel histológico, se observó la desorganización de las fibras del ligamento periodontal en la zona de compresión y la elongación de éstas en el área de tensión. Además, se verificó la presencia de múltiples lagunas de reabsorción ósea y la aparición de células multinucleadas compatibles con osteoclastos en la zona de compresión. Estos antecedentes nos permiten concluir que el modelo de movilización dentaria utilizado en este caso fue efectivo en lograr un desplazamiento dentario comparable al de estudios previos ${ }^{(21,22,23)}$.

La TRAP corresponde a marcador para osteoclastos ${ }^{(18)}$ y ha sido utilizada en diferentes estudios de movilización dentaria, donde se ha verificado la presencia de células TRAP positivas en el área de presión asociadas a lagunas de reabsorción ósea ${ }^{(22,23)}$. En modelos de movimiento dentario en animales se ha observado que la expresión de TRAP alcanza un nivel máximo en el día 3 posterior a la aplicación de fuerzas ${ }^{(24)}$. Luego de esto, la producción de esta enzima tiende a disminuir de manera significativa en el día $7^{(24)}$. Esta descripción es coincidente con la diferenciación osteoclástica, que alcanza su máximo nivel entre los días 3 y 5 luego de la aplicación de fuerzas $^{(24)}$. Estos antecedentes concuerdan con nuestros resultados, en los cuales pudimos observar que los mayores niveles de expresión de TRAP ocurrieron en la zona de compresión en el día 3, posterior a la aplicación de la fuerza de movilización dentaria, observándose luego una disminución en la marca para TRAP en los días 5 y 7 . En el día 5 se apreció una marca menor a la esperada, respecto del control, probablemente por defectos de la técnica o del procesamiento de la muestra. En el área de tensión se observó una baja reacción para TRAP, lo que se explica por la remodelación fisiológica y la migración distal que posee la especie en estudio ${ }^{(25)}$.

Utilizando inmunohistoquímica se pudo observarque la producción de MT1-MMP se encuentra asociada a fibroblastos de la zona de compresión del ligamento periodontal y osteoclastos involucrados en la degradación de tejido óseo de la misma región. No se logró observar la producción de MT1MMP en la zona de tensión del ligamento periodontal. Estudios previos han permitido identificar la producción de MT1-MMP en fibroblastos de piel y encía, macrófagos infiltrantes de placas ateromatosas, células tumorales y osteoclastos ${ }^{(26,27)}$. La expresión de MT1-MMP ha sido localizada en los podosomas de osteoclastos, lo que ha permitido vincular la actividad de esta enzima con la migración celular ${ }^{(27)}$. Dentro de los sustratos que podría degradar la MT1-MMP asociados a la migración encontramos a colágeno tipo I y el proteoglicano CD-44(13). En células tumorales la degradación de CD-44 desde la superficie celular puede potenciar la migración e invasión tumoral ${ }^{(13)}$. Estos datos permiten argumentar que la degradación de estas macromoléculas por MT1-MMP podría estar potenciando positivamente la migración de células clásticas a través de la matriz ósea.

La deficiencia de MT1-MMP en ratones induce un incremento en la producción de TRAP y en el número de osteoclastos. Además, el tejido óseo de estos animales manifiesta marcados signos de osteopenia ${ }^{(16)}$ Uno de los reguladores claves de la diferenciación osteoclástica es la citoquina Activador del Receptor de Ligando de NF-kB (RANKL)(28). Esta citoquina es una proteína de transmembrana cuya actividad biológica puede ser regulada a través de su procesamiento proteolítico y liberación desde la membrana celular ${ }^{(29)}$. Utilizando cultivos primarios de osteoblastos y osteoclastos se ha observado que la MT1-MMP podría participar regulando negativamente la osteoclastogénesis a través del procesamiento proteolítico y la aparente inactivación de la citoquina RANKL ${ }^{(29)}$. Estos antecedentes permiten proponer que la producción de MT1-MMP en osteoclastos podría estar controlando negativamente el fenómeno de osteoclastogénesis durante el movimiento dentario.

La reabsorción ósea, tanto in vitro como in vivo, es estimulada por TNF- $\alpha$, una citoquina pro-inflamatoria producida principalmente por monocitos, macrófagos, fibroblastos y también por osteoblastos ${ }^{(30)}$ Estudios en humanos han evidenciado un aumento en los niveles de TNF- $\alpha$ en el fluido gingival crevicular durante el movimiento dentario y se ha demostrado en modelos animales que TNF- $\alpha$ juega un papel importante en la movilización dentaria en una etapa tardía( ${ }^{(30)}$. Es destacable que estudios recientes han demostrado que la MT1-MMP puede regular la actividad biológica de TNF- $\alpha$ a través de su procesamiento proteolítico ${ }^{(14)}$. Sin embargo, no está claro si esta modificación proteolítica ocasiona una activación o inhibición de la mencionada citoquina. Es interesante destacar que una de las citoquinas que estimulan la producción de MT1-MMP es TNF- $\alpha^{(31)}$, y a través de este mecanismo se podría estar potenciando de manera autocrina la producción de esta enzima proteolítica. Estudios futuros deberán comprobar si es que TNF- $\alpha$ está regulando la producción de MT1-MMP durante la movilización dentaria.

Los resultados obtenidos en el presente estudio nos permiten proponer que TRAP y MT1-MMP se expresan en los tejidos periodontales durante la movilización dentaria en el modelo utilizado y que participarían de los fenómenos de adaptación y/o remodelación tisular durante este proceso.

\section{AGRADECIMIENTOS}

Queremos dedicar este trabajo en memoria y agradecimiento al Sr. Juan Méndez Pradine (QEPD), quien trabajó dedicadamente en el procesamiento histológico de las muestras de este estudio. El presente trabajo fue financiado por el proyecto FONDECYT 1061065 (PS) y constituyó el trabajo de investigación que desarrolló Paulina Rey Droghetti para obtener el título de Cirujano Dentista en la Facultad de Odontología, Universidad de Chile. 


\section{REFERENCIAS BIBLIOGRÁFICAS}

1. Melsen B. Tisue reaction to orthodontic tooth movement - a new paradigm. Eur J Orthod, 2001; 23: 671-681.

2. Tsay TP, Chen MH, Oyen OJ. Osteoclast activation and recruitment after application of orthodontic force. Am J Orthod Dentofacial Orthop, 1999; 115: 323-330.

3. Rody WJ Jr, King GJ, Gu G. Osteoclast recruitment to sites of compression in orthodontic tooth movement. Am J Orthod Dentofacial Orthop, 2001; 120: 477-489.

4. Krane SM, Inada M. Matrix metalloproteinases and bone. Bone, 2008; 43: 7-18.

5. Parks WC, Wilson CL, López-Boado YS. Matrix metalloproteinases as modulators of inflammation and innate immunity. Nat Rev Immunol, 2004: 617-629.

6. Domon S, Shimokawa H, Matsumoto Y, Yamaguchi S, Soma K. In situ hybridization for matrix metalloproteinase-1 and cathepsin $\mathrm{K}$ in rat rootresorbing tissue induced by tooth movement. Arch Oral Biol, 1999; 44: 907-915.

7. Beertsen W, Holmbeck K, Niehof A, Bianco P, Chrysovergis K, BirkedalHansen H, Everts V. On the role of MT1-MMP, a matrix metalloproteinase essential to collagen remodelling, in murine molar eruption and root growth. Eur J Oral Sci, 2002; 110: 445-451.

8. Ingman $T$, Apajalahti $S$, Mäntylä $P$, Savolainen $P$, Sorsa T. Matrix metalloproteinase -1 and -8 in gingival crevicular fluid during orthodontic tooth movement: a pilot study during 1 month of follow-up after fixed appliance activation. Eur J Orthod, 2005; 27: 202-207.

9. Takahashi I, Nishimura M, Onodera K, Bae JW, Mitani H, Okazaki M, Sasano Y, Mitani H. Expression of MMP-8 and MMP-13 genes in the periodontal ligament during tooth movement in rats. J Dent Res, 2003; 82: 646-651.

10. Takahashi I, Onodera K, Nishimura M, Mitnai H, Sasano $Y$, Mitani $\mathrm{H}$. Expression of genes for gelatinases and tissue inhibitors of metalloproteinases in periodontal tissues during orthodontic tooth movement. J Mol Histol, 2006; 37: 333-342.

11. McCulloch CA, Lekic P, McKee MD. Role of physical forces in regulating the form and function of the periodontal ligament. Periodontol 2000, 2000; 24: 56-72.

12. Tsubota M, Sasano $\mathrm{Y}$, Takahashi I, Kagayama M, Shimauchi $\mathrm{H}$. Expression of MMP-8 and MMP-13 mRNAs in rat periodontium during tooth eruption. J Dent Res, 2002; 81: 673-678.

13. Holmbeck K, Bianco P, Yamada S, Birkedal-Hansen H. MT1-MMP: a tethered collagenase. J Cell Physiol, 2004; 200: 11-19.

14. Tam EM, Morrison CJ, Wu YI, Stack MS, Overall CM. Membrane protease proteomics: Isotope-coded affinity tag MS identification of undescribed MT1-matrix metalloproteinase substrates. Proc Natl Acad Sci, USA 2004; 101: 6917-6922.

15. Oyarzún AD, Arancibia R, Hidalgo R, Peñafiel C, Cáceres M, González MJ, Martínez J, Smith PC. Involvement of MT1-MMP and TIMP-2 in human periodontal disease. Oral Diseases, 2010; 16: 388-395.

16. Holmbeck K, Bianco P, Caterina J, Yamada S, Kromer M, Kuznetsov SA, Mankani M, Robey PG, Poole AR, Pidoux I, Ward JM, BirkedalHansen H. MT1-MMP-deficient mice develop dwarfism, osteopenia, arthritis, and connective tissue disease due to inadequate collagen turnover. Cell, 1999; 99: 81-92.

17. Boyce BF, Yao Z, Zhang Q, Guo R, Lu Y, Schwarz EM, Xing L. New roles for osteoclasts in bone. Ann NY Acad Sci, 2007; 1116: 245-254.
18. Cole AA, Walters LM. Tartrate-resistant acid phosphatase in bone and cartilage following decalcification and cold embedding in plastic. $J$ Histochem Cytochem, 1987; 35: 203-206.

19. Linsuwanont-Santiwong B, TakagiY, OhyaK, Shimokawa H. Expression of MT1-MMP during deciduous tooth resorption in odontoclasts. J Bone Miner Metab, 2006; 24: 447-453.

20. Dong Z, Bonfil RD, Chinni S, Deng X, Trindade Filho JC, Bernardo M, Vaishampayan U, Che M, Sloane BF, Sheng S, Fridman R, Cher ML. Matrix metalloproteinase activity and osteoclasts in experimental prostate cancer bone metastasis tissue. Am J Pathol, 2005; 166: 1173-1186.

21. Kobayashi H, Ochi K, Saito I, Hanada K, Maeda T. Alterations in ultraestructural localization of growth-associated Protein-43 (GAP-43) in periodontal ruffini endings of rat molars during experimental tooth movement. J Dent Res, 1998; 77: 503-517.

22. Bonafe-Oliveira L, Faltin RM, Arana-Chavez VE. Ultraestructural and histochemical examination of alveolar bone at the pressute areas of rat molars submitted to continuous orthodontic force. Eur J Oral Sci, 2003; 111: 410-416.

23. Kawarizadeh A, Bourauel C, Zhang D, Götz W, Jäger A. Correlation of stress and strain profiles and the distribution ofosteoclastic cells induced by orthodontic loading in rat. Eur J Oral Sci, 2004; 112: 140-147.

24. Gu G, Lemmery S, King G. Effect of appliance reactivation after decay of initial activation on osteoclasts, tooth movement, and root resorption. Angle Orthod, 1999; 69: 515-522.

25. Ren Y, Maltha JC, Kuijpers-Jagtman AM. The rat as a model for orthodontic tooth movement - a critical review and a proposed solution. Eur J Orthod, 2004; 26: 483-490.

26. Sato T, del Carmen Ovejero M, Hou P, Heegaard AM, Kumegawa $M$, Foged NT, Delaissé JM. Identification of the membrane-type metalloproteinase MT1-MMP in osteoclast. J Cell Sci, 1997; 110 (Pt 5): 589-596.

27. Rajavashisth TB, Xu XP, Jovinge $S$, Meisel $S$, Xu XO, Chai NN Fishbein MC, Kaul S, Cercek B, Sharifi B, Shah PK. Membrane type 1 matrix metalloproteinase expression in human atherosclerotic plaques: evidence for activation by proinflammatory mediators. Circulation, 1999; 99: 3103-3109.

28. Camozzi V, Vescini F, Luisetto G, Moro L. Bone organic matrix components: their roles in skeletal physiology. J Endocrinol Invest, 2010; 33: 13-15.

29. Hikita A, Yana I, Wakeyama H, Nakamura M, Kadono Y, Oshima Y Nakamura K, Seiki M, Tanaka S. Negative regulation of osteoclastogenesis by ectodomain shedding of receptor activator of NF-kappaB ligand. J Biol Chem, 2006; 281: 36846-36855.

30. Zhang D, Goetz W, Braumann B, Bourauel C, Jaeger A. Effect of soluble receptors to interleukin-1 and tumor necrosis factor alpha on experimentally induced root reserption in rats. J Periodont Res, 2003; 38: 324-332

31. Smith PC, Guerrero J, Tobar N, Cáceres M, González MJ, Martínez J. Tumor necrosis factor-alpha-stimulated membrane type 1-matrix metalloproteinase production is modulated by epidermal growth factor receptor signaling in human gingival fibroblasts. J Periodontal Res, 2009; 44: 73-80. 